\title{
Finite-amplitude ferro-convection and electro-convection in a rotating fluid
}

\author{
Pradeep Ganapathi Siddheshwar ${ }^{1} \cdot$ Om Paramsukh Suthar ${ }^{2} \cdot$ Kanchana Chinnaswamy $^{3}$
}

Received: 1 June 2019 / Accepted: 18 October 2019 / Published online: 4 November 2019

(c) Springer Nature Switzerland AG 2019

\begin{abstract}
The onset of Rayleigh-Bénard convection in a horizontal ferrofluid layer subjected to rotation with respect to the vertical axis is theoretically investigated in the paper. Using a truncated Fourier series representation, an analytically intractable fifth-order Lorenz model that has two quadratic nonlinearities is derived and then reduced to the analytically tractable Landau equation with cubic nonlinearity using the method of multi-scales. The critical Rayleigh number of the linear stability theory and that of the energy stability method is drawn from other works and compared with that obtained by the weakly nonlinear stability method reported in the paper. It is found that the critical Rayleigh number obtained by the two nonlinear theories predicts subcritical motions. Further, the results on electro-convection in a rotating fluid layer are extracted from the corresponding problem of ferro-convection by establishing an one-to-one correspondence between the governing equations of the two problems.
\end{abstract}

Keywords Ferro-convection · Electro-convection · Energy stability · Rayleigh-Bénard · Lorenz model

\section{Introduction}

The theory of rotating Newtonian fluids and Rayleigh-Bénard convection in such fluids is a well-studied topic [1-6]. The corresponding problem in ferrofluids (called rotating ferro-convection) and in dielectric fluids (called rotating electro-convection) can be interesting for reasons more than one.

Ferrofluids are synthesized suspensions of micron-sized magnetic particles in a carrier liquid of suitable choice. The carrier liquids generally used are synthetic oil, water, kerosene, or such other liquids. The suspended magnetic particles that possess a fixed magnetic moment are coated with a surfactant, such as oleic acid, to avoid coagulation. In the presence of an external magnetic field, the resulting orientation of the magnetic particles leads to a net magnetization of the fluid which depends on both the applied magnetic field and the temperature of the fluid. The applied magnetic field exerts a force on the fluid which is known as the Kelvin force. When the magnetic field strength is very high, the magnetization achieves its saturation value and the particles get aligned with the applied field. The magnetization generally depends on the magnetic field, temperature, and density of the ferrofluid. Hence, a local variation in magnetic field/temperature may give rise to thermo-magnetic convection which is equivalent to the natural convection occurring due to variation in temperature only. This phenomenon has attracted the interest of many researchers in past five decades and has widespread scientific applications [7-15].

The linear and nonlinear ferro-convection in nonrotating fluids occupying a very shallow enclosure has now been extensively studied [16-39]. The linear and nonlinear problems of rotating ferro-convection have also attracted

$\triangle$ Pradeep Ganapathi Siddheshwar, mathdrpgs@gmail.com; Om Paramsukh Suthar, ompsuthar.maths@mnit.ac.in; Kanchana Chinnaswamy, kanchana@hit.edu.cn | 'Department of Mathematics, Bangalore University, Jnanabharathi Campus, Bangalore 560056, India. ${ }^{2}$ Department of Mathematics, Malaviya National Institute of Technology Jaipur, Jaipur 302017, India. ${ }^{3}$ College of Science, Harbin Institute of Technology, Shenzhen, Nanshan District, Guangdong province, Shenzhen 518055, China. 
attention of many [40-45]. The nonlinear stability analysis of rotating ferro-convection reported above has been studied using weakly nonlinear stability analysis (based on Lorenz model or Ginzburg-Landau equation) or by energy stability analysis.

The work on nonlinear, rotating electro-convection is nonexistent, though there are few works on nonlinear electro-convection in the absence of rotation (Siddheshwar and Radhakrishna [46] and reference therein). The one-to-one correspondence between the linear problems of ferro-convection and electro-convection lead Siddheshwar $[47,48]$ to take a unified approach at studying both.

The exhaustive literature survey on ferro-convection and electro-convection reveals that the following aspects are as yet unconsidered:

1. Recognizing that linear and nonlinear electro-convection problems in rotating and nonrotating systems have an analogy with the corresponding problems of ferro-convection.

2. Comparing the critical Rayleigh numbers of linear stability, weakly nonlinear stability, and energy stability of rotating ferro-convection as well as rotating electroconvection - existence of subcritical motions.

3. Connecting the analytically intractable Lorenz model of nonrotating and rotating ferro-convection with the corresponding Landau model that is analytically tractable.

4. Analyzing whether the analytical solution of the Landau equation provides insights into the nature of the amplitude of convection in transient and steady-state regimes.

The objective of the present paper is to consider the aforementioned aspects that concern nonlinear rotating ferro-convection (and corresponding electro-convection problem by analogy).

\section{Problem formulation for the rotating ferro-convection}

Consider a horizontal layer of ferrofluid confined between two infinitely extended parallel plates, $z=0$ and $z=d$. We choose the right-hand system of coordinates $(x, y, z)$, where $z$-axis is assumed in such a way that the gravitational force, $\mathbf{g}$, is taken as $\mathbf{g}=-g_{0} \hat{\mathbf{k}}$. The layer is subjected to rotation with respect to the $z$-axis with a constant angular speed $\boldsymbol{\Omega}$. The motion described here occurs in a way as it appears to an observer at rest in a frame rotating about the same axis and with the same angular velocity (uniformly rotating frame of reference). An external magnetic field $\mathbf{H}$ is applied in the $z$-direction. An adverse temperature gradient across the fluid layer is maintained by imposing a uniform temperature difference $\Delta T$ between the plates. Schematic of the same is described in Fig. 1.

For mathematical tractability, all physical quantities are assumed to be independent of the horizontal co-ordinate, $y$. Thus, the study pertains to two-dimensional ferro-convection confined within a horizontal ferrofluid layer. The height, $d$, and breadth, $b$, are such that when $d / b<<1$, and hence, there is no effect of the lateral walls $(x)$ on the dynamics in the bulk of the ferromagnetic fluid.

\subsection{Governing equations}

To incorporate the effect of uniform rotation into the equation of conservation of linear momentum, the acceleration term can be taken as

$\left(\frac{\mathrm{Dq}}{\mathrm{D} t}\right)_{l}=\left(\frac{\mathrm{Dq}}{\mathrm{D} t}\right)_{R}+\boldsymbol{\Omega} \times(\boldsymbol{\Omega} \times \mathbf{r})-2(\mathbf{q} \times \mathbf{\Omega})_{R^{\prime}}$

where q denotes the velocity vector and $t$ is time. The subscripts $/$ and $R$ refer to inertial and rotating frames of reference and $\mathbf{r}$ is the radius vector. The operator $\frac{D}{D t}$ in Eq. (1) is given by $\frac{\mathrm{D}}{\mathrm{D} t}=\frac{\partial}{\partial t}+\mathbf{q} \cdot \boldsymbol{\nabla}$. The second and third terms in Eq. (1) are centrifugal and Coriolis forces that occur due to rotation. Since the rotation is uniform, the Euler force is neglected in Eq. (1). The centrifugal force can be expressed as the gradient of a scalar quantity:

$\mathbf{\Omega} \times(\boldsymbol{\Omega} \times \mathbf{r})=-\boldsymbol{\nabla}\left(\frac{1}{2}|\mathbf{\Omega}|^{2}|\mathbf{r}|^{2}\right)$,

where $|\mathbf{r}|$ is the distance from the axis of rotation. Thus, the governing equations for studying the rotating ferroconvection problem with applied magnetic field after omitting subscript $R$ are

$\boldsymbol{\nabla} \cdot \mathbf{q}=0$,

$\rho_{0} \frac{\partial \mathbf{q}}{\partial t}=-\nabla P+\rho(T) \mathbf{g}+\mu_{0}(\mathbf{M} \cdot \boldsymbol{\nabla}) \mathbf{H}+\mu \nabla^{2} \mathbf{q}$

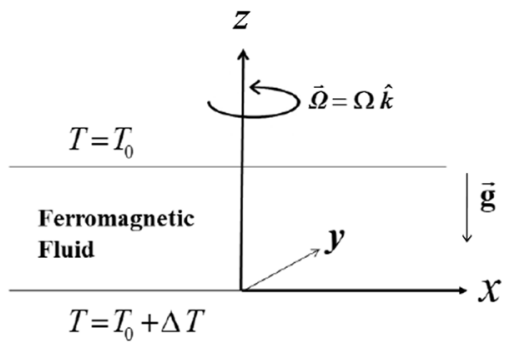

Fig. 1 Schematic of the ferro-convection problem 
$\frac{\partial T}{\partial t}+(\mathbf{q} \cdot \boldsymbol{\nabla}) T=\kappa \nabla^{2} T$,

We are considering only small-scale convective motion and hence $|(\mathbf{q} \cdot \boldsymbol{\nabla}) \mathbf{q}|<<1$. The centrifugal force in Eq. (4) is absorbed into the pressure gradient term. Thus, pressure, $P$, can be written as $P=p-\frac{\rho_{0}}{2}|\Omega|^{2}|\mathbf{r}|^{2}$, the sum of the hydro-static pressure $(p)$ and that due to the centrifugal force. The physical quantities $\rho_{0}, \mu_{0}, \mu, \kappa, \mathbf{M}, \mathbf{H}$, and $T$ represent the static density, magnetic permeability, effective viscosity, thermal diffusivity, magnetization, magnetic field, and temperature, respectively. In writing Eq. (4), the Boussinesq approximation is assumed to be valid. The state equation may be written as

$\rho(T)=\rho_{0}\left[1-\alpha\left(T-T_{0}\right)\right]$,

where $\alpha$ is the thermal expansion coefficient and $T_{0}$ is the reference temperature. We presume the ferrofluid to be electrically insulating and write the following Maxwell's equations for the magnetic field, $\mathbf{H}$, and the magnetic induction, $\mathbf{B}$, as

$\boldsymbol{\nabla} \times \mathbf{H}=\mathbf{0}, \quad \boldsymbol{\nabla} \cdot \mathbf{B}=\mathbf{0}$.

Furthermore, the relationship between these fields is

$\mathbf{B} \equiv \mu_{0}(\mathbf{H}+\mathbf{M})$,

where $\mathbf{M}=M(T, H) \hat{\mathbf{H}}$. The magnetic equation of state is given by (Finlayson [16])

$M(T, H)=M_{0}-K\left(T-T_{0}\right)+\chi\left(H-H_{0}\right)$,

where $K$ and $\chi$ are the pyromagnetic and magnetic susceptibility coefficients, $M_{0}=M\left(T_{0}, H_{0}\right)$ is the reference magnetization, and $H_{0} \hat{\mathbf{k}}$ is the applied uniform vertical magnetic field.

We note from Eq. (7), that we can write $\mathbf{H}=\boldsymbol{\nabla} \phi$, where $\phi$ is the magnetic potential.

\subsection{Boundary conditions}

We consider the boundaries to be such that the tangential stress vanishes. To maintain a static temperature difference across the layer, we keep the lower boundary $(z=0)$ at a temperature $T_{0}+\Delta T(\Delta T>0)$ and the upper boundary $(z=d)$ at a temperature $T_{0}$. We, therefore, assume stressfree and isothermal boundary conditions:

$$
\left.\begin{array}{r}
w=\frac{\partial^{2} w}{\partial z^{2}}=0, T=T_{0}+\Delta T \text { at } z=0 \\
w=\frac{\partial^{2} w}{\partial z^{2}}=0, T=T_{0} \text { at } z=d
\end{array}\right\},
$$

where $w$ is the $z$-components of velocity vector, $\mathbf{q}$.
To specify the magnetic potential boundary condition, we assume continuity of the tangential component of the magnetic field and the normal component of the magnetic induction across the boundary. To derive magnetic potential boundary condition, we considered general boundary condition for the perturbed magnetic potential, $\phi$ :

$\left.\begin{array}{l}D \phi+\frac{k \phi}{1+\chi}-T=0 \text { at } z=0 \\ D \phi-\frac{k \phi}{1+\chi}-T=0 \text { at } z=d\end{array}\right\}$

where $D=\frac{d}{d z}$ and $k$ is dimensionless wave number. Since isothermal boundary condition is assumed for temperature and when $\chi \rightarrow \infty$ at the boundary, we obtain $D \phi=0$ (see Finlayson [16]) at $z=0$ and $d$.

There are umpteen number of instances in which idealized boundary conditions have been considered with the sole purpose of seeking qualitative results (see Finlayson [16], Suslov [51], Laroze et al. [38], Rahman and Suslov [39], and certain references therein). The present paper is one of them. The analysis is exact and trustworthy in this case. We, however, note that the problem is best done with realistic boundary condition but the price we pay for this is that the analysis, especially the nonlinear one, is fraught with errors due to mandatory numerical procedure imposed by it. This study is at the present time is contemplated to be completed in the nearest future.

\subsection{Basic state solution}

Using boundary conditions (10) and (11), the conductive basic quiescent state solution may be written as

$\mathbf{q}_{b}=\mathbf{0}$,

$T_{b}(z)=T_{0}-\beta(z-d)$,

$\mathbf{H}_{b}(z)=\left(H_{0}-\frac{K \beta(z-d)}{1+\chi}\right) \hat{\mathbf{k}}$,

$\mathbf{M}_{b}(z)=\left(M_{0}+\frac{K \beta(z-d)}{1+\chi}\right) \hat{\mathbf{k}}$,

where $\beta=\Delta T / d$ and the subscript $b$ represents the basic state.

\section{Stability analysis}

To perform stability analysis, we introduce perturbations to the basic state in the form 


$$
\left.\begin{array}{r}
\mathbf{q}=\mathbf{0}+\mathbf{q}^{\prime}, \quad \mathbf{H}=\mathbf{H}_{b}+\mathbf{H}^{\prime}, \quad \mathbf{M}=\mathbf{M}_{b}+\mathbf{M}^{\prime}, \\
\rho=\rho_{0}+\rho^{\prime}, \quad P=P_{b}+P^{\prime}, \quad T=T_{b}+T^{\prime},
\end{array}\right\}
$$

where the prime denotes perturbed quantities.

\subsection{Perturbation equations}

Using Eqs. (9) and (16) in Eq. (8), we get

$$
\left.\begin{array}{l}
H_{x}^{\prime}+M_{x}^{\prime}=\left(1+\frac{M_{0}}{H_{0}}\right) H_{x^{\prime}}^{\prime} \\
H_{z}^{\prime}+M_{z}^{\prime}=(1+\chi) H_{z}^{\prime}-K T^{\prime}
\end{array}\right\} .
$$

Imposing perturbation on governing equations (3)-(5), we get the perturbed governing equations in the following form:

$\boldsymbol{\nabla} \cdot \mathbf{q}^{\prime}=0$,

$$
\begin{aligned}
& \rho_{0} \frac{\partial \mathbf{q}^{\prime}}{\partial t}=-\nabla P_{\text {eff }}+\rho_{0} \alpha T^{\prime} g \hat{\mathbf{k}}+\mu \boldsymbol{\nabla}^{2} \mathbf{q}^{\prime}+2 \rho_{0}\left(\mathbf{q}^{\prime} \times \mathbf{\Omega}\right) \\
& \quad+\mu_{0}\left[\boldsymbol{\nabla}\left(\phi_{z}^{\prime} M_{b}\right)-\frac{K \beta\left\{(1+\chi) \phi_{z}^{\prime}-K T^{\prime}\right\} \hat{\mathbf{k}}}{1+\chi}-K T^{\prime} \boldsymbol{\nabla} \phi_{z}^{\prime}\right],
\end{aligned}
$$

$\frac{\partial T^{\prime}}{\partial t}+\left(\mathbf{q}^{\prime} \cdot \boldsymbol{\nabla}\right) T^{\prime}=\kappa \nabla^{2} T^{\prime}+\beta w^{\prime}$.

where $P_{\text {eff }}=P-\frac{\mu_{0} M_{0}}{2 H_{0}} \phi_{x}^{\prime 2}-\frac{\mu_{0} \chi}{2} \phi_{z}^{\prime 2}, w^{\prime}$ is the perturbed velocity in $z$-direction, and $\phi_{z}$ is the differentiation of $\phi$ with respect to $z$.

\subsection{Nondimensionalization}

To convert the governing equations to dimensionless form, we introduce the characteristic scales $d$ for length, $d^{2} / \kappa$ for time, $\kappa / d$ for velocity, $\beta d$ for temperature, $\beta d^{2} K /(1+\chi)$ for magnetic scalar potential, and $\mu \kappa / d^{2}$ for the pressure and the stress tensor. Using these scales in Eqs. (18)-(20) and simplifying, we get the dimensionless governing equations for the perturbations in the following form:

$\boldsymbol{\nabla} \cdot \mathbf{q}=0$,

$$
\begin{aligned}
\frac{1}{\operatorname{Pr}} \frac{\partial \mathbf{q}}{\partial t} & =-\nabla P+\operatorname{Ra}\left(1+M_{1}\right) \theta \hat{k}-\operatorname{Ra} M_{1} \phi_{z} \hat{\mathbf{k}} \\
& +\nabla^{2} \mathbf{q}+\sqrt{\operatorname{Ta}}(\mathbf{q} \times \hat{\mathbf{k}})-\operatorname{Ra} M_{1} \theta \boldsymbol{\nabla} \phi_{z^{\prime}}
\end{aligned}
$$

$\frac{\partial \theta}{\partial t}+(\mathbf{q} \cdot \boldsymbol{\nabla}) \theta=\nabla^{2} \theta+w$,
$M_{3} \frac{\partial^{2} \phi}{\partial x^{2}}+\frac{\partial^{2} \phi}{\partial z^{2}}-\frac{\partial \theta}{\partial z}=0$

The nondimensional parameters in Eqs. (22) and (24) are $\operatorname{Pr}=\frac{\mu}{\rho_{0} \kappa}$, Prandtl number, $\mathrm{Ra}=\frac{\rho_{0} \alpha \Delta T d^{3} g}{\mu \kappa}$, Rayleigh number, $\mathrm{Ta}=\left(\frac{2 d^{2} \rho_{0} \Omega}{\mu}\right)^{2}$, Taylor number, $M_{1}=\frac{\mu_{0} K^{2} \Delta T}{g \rho_{0}(1+\chi) d^{\prime}}$ ratio of the magnetic and buoyancy forces, and $M_{3}=\frac{1+M_{0} / H_{0}}{1+\chi}$, non-buoyancy magnetization parameter. If we take $M_{1}=0$, then that corresponds to the nonmagnetic Rayleigh-Bénard convection problem. The term $M_{3}$ measures the departure of linearity in the magnetic equation of state defined in Eq. (9) and $M_{3}=1$ corresponds to linear magnetization.

We operate curl on Eq. (22) to get the vorticity equation:

$\frac{1}{\operatorname{Pr}} \frac{\partial \zeta}{\partial t}=\nabla^{2} \zeta+\sqrt{\operatorname{Ta}} \frac{\partial w}{\partial z}-\operatorname{Ra} M_{1} \frac{\partial\left(\theta, \phi_{z}\right)}{\partial(x, z)}$

where $\zeta=[\nabla \times \mathbf{q}]_{z}$ is the $z$-component of vorticity which describes the local spinning motion of the continuum point. The term, $\frac{\partial\left(\theta, \phi_{z}\right)}{\partial(x, z)}$, represents the Jacobian term and is defined as

$\frac{\partial\left(\theta, \phi_{z}\right)}{\partial(x, z)}=\frac{\partial \theta}{\partial x} \frac{\partial \phi_{z}}{\partial z}-\frac{\partial \theta}{\partial z} \frac{\partial \phi_{z}}{\partial x}$.

Operating $\hat{\mathbf{k}} \cdot$ curl curl on Eq. (22) results in the following equation:

$$
\begin{aligned}
\frac{1}{\operatorname{Pr}} \frac{\partial}{\partial t}\left(\nabla^{2} w\right) & =\nabla^{4} w-\sqrt{\operatorname{Ta}} \frac{\partial \zeta}{\partial z}+\operatorname{Ra}\left(1+M_{1}\right) \nabla_{1}^{2} \theta-\operatorname{Ra} M_{1} \nabla_{1}^{2} \phi_{z} \\
& -\operatorname{Ra} M_{1}\left[\frac{\partial}{\partial x} \frac{\partial\left(\theta, \phi_{z}\right)}{\partial(x, z)}\right]
\end{aligned}
$$

where $\nabla_{1}^{2}=\frac{\partial^{2}}{\partial x^{2}}$. The system of Eqs. (23)-(26) are solved subject to the boundary condition in nondimensional form:

$w=D^{2} w=\theta=D \zeta=D \phi=0$ at $z=0,1$.

This boundary condition, though unrealistic, facilitates an analytical solution that gives qualitatively similar results to that of realistic boundaries.

\section{Lorenz model}

We assume the following truncated Fourier series solutions for $u, v, w, \theta$, and $\phi$ : 


$$
\begin{aligned}
u(x, z, t) & =-A_{1}(t) \pi \sin (k x) \cos (\pi z) \\
w(x, z, t) & =k A_{1}(t) \cos (k x) \sin (\pi z) \\
v(x, z, t) & =B_{1}(t) \cos (k x) \cos (\pi z)+B_{2}(t) \cos (2 k x) \\
\theta(x, z, t) & =C_{1}(t) \cos (k x) \sin (\pi z)+C_{2}(t) \sin (2 \pi z) \\
\phi(x, z, t) & =D_{1}(t) \cos (k x) \cos (\pi z)+D_{2}(t) \cos (2 \pi z)
\end{aligned}
$$

where $A_{1}(t), B_{1}(t), B_{2}(t), C_{1}(t), C_{2}(t), D_{1}(t)$ and $D_{2}(t)$ are amplitudes. Substituting Eq. (28) in Eq. (24) yields $D_{1}$ and $D_{2}$ in terms of $C_{1}$ and $C_{2}$, respectively:

$D_{1}(t)=-\frac{\pi}{\delta_{M}^{2}} C_{1}(t) \quad D_{2}(t)=-\frac{1}{2 \pi} C_{2}(t)$,

where $\delta_{M}^{2}=\left(\pi^{2}+M_{3} k^{2}\right)$. On substituting Eq. (28) in Eqs. (25) and (26) and using the slave relationship (29) in one of the equations and the orthogonality conditions, we get the following system of equations

$$
\left.\begin{array}{rl}
\dot{A}_{1}(t)= & -\delta^{2} \operatorname{Pr} A_{1}(t)-\frac{\pi \operatorname{Pr} \sqrt{\mathrm{Ta}}}{\delta^{2}} B_{1}(t) \\
& +k \operatorname{Pr} \operatorname{Ra} C_{1}(t)\left[\xi_{1}-\xi_{2} C_{2}(t)\right], \\
\dot{B}_{1}(t)= & A_{1}(t) \pi\left(\operatorname{Pr} \sqrt{\mathrm{Ta}}-k B_{2}(t)\right)-\delta^{2} \operatorname{Pr} B_{1}(t), \\
\dot{B}_{2}(t)= & -4 k^{2} \operatorname{Pr} B_{2}(t)-\frac{\pi k}{2} A_{1}(t) B_{1}(t), \\
\dot{C}_{1}(t)= & A_{1}(t)\left(\pi k C_{2}(t)+k\right)-\delta^{2} C_{1}(t), \\
\dot{C}_{2}(t)= & -\frac{\pi k}{2} A_{1}(t) C_{1}(t)-4 \pi^{2} C_{2}(t),
\end{array}\right\}
$$

where an over dot on amplitudes denotes derivative with respect to $t, \delta^{2}=k^{2}+\pi^{2}, \xi_{1}=\frac{\delta_{M}^{2}+k^{2} M_{1} M_{3}}{\delta^{2} \delta_{M}^{2}}$, and $\xi_{2}=\frac{\pi k^{2} M_{1} M_{3}}{\delta^{2} \delta_{M}^{2}}$

\section{Steady finite-amplitude convection and subcritical motions}

Assuming the amplitudes to be independent of time in the Lorenz system (30), we get the governing equations for the study of steady two-dimensional finite-amplitude convection in the form of a coupled system of algebraic equations which is then reduced to a cubic equation in $A_{1}^{2}$ :

$a_{1}\left(A_{1}^{2}\right)^{3}+a_{2}\left(A_{1}^{2}\right)^{2}+a_{3} A_{1}^{2}+a_{4}=0$.
The expressions for $a_{i}^{\prime} \mathrm{s}, i=1,2,3,4$, are quite lengthy and have been omitted for brevity. The minimum value of $\mathrm{Ra}$ for which the discriminant of Eq. (31) vanishes gives us the steady, finite-amplitude Rayleigh number $\left(\mathrm{Ra}_{\mathrm{f}}\right)$. For the present problem, a polynomial of degree nine in Ra was obtained by setting the discriminant of the above equation to be zero and thus $\mathrm{Ra}_{\mathrm{f}}$ had to be evaluated numerically together with the wave number, $k$, as a function of $\mathrm{Ta}, M_{1}, M_{3}$, and $\mathrm{Pr}$.

With the intention of comparing the results obtained in the present study with those of the linear stability and the energy stability ones, we recollect that for the present problem the linear stability Rayleigh number is given by Gupta and Gupta [40]

$\mathrm{Ra}_{\mathrm{sF}}=\frac{\delta_{M}^{2}\left(\delta^{6}+\pi^{2} \mathrm{Ta}\right)}{k^{2}\left(k^{2} M_{1} M_{3}+\delta_{M}^{2}\right)}$

and the energy stability Rayleigh number (as reported in [35]) is given by

$\mathrm{Ra}_{\mathrm{eF}}=\frac{\left[4 \delta_{M}^{2}-\frac{\pi^{2} M_{1}}{\left(1+M_{1}\right)}\right]\left(\delta^{6}+\pi^{2} \mathrm{Ta}\right)}{4 k^{2}\left(1+M_{1}\right) \delta_{M}^{2}-2 \pi^{2} M_{1}}$.

Equation (33) is Eq. (75) in Sunil and Mahajan [43] but with the notations used in the present paper. In the next section, we reduce the fifth-order Lorenz system into a first-order Landau equation by using the method of multi-scales.

\section{Landau amplitude equation for small amplitude convective motion}

We assume a slow time scale $\tau=\epsilon^{2} t$ and use the following regular perturbation expansion as done by Siddheshwar and Kanchana [48] for the amplitudes and also for Ra appearing in the system of Eq. (30):

$\left[\begin{array}{c}A_{1} \\ B_{1} \\ B_{2} \\ C_{1} \\ C_{2} \\ \mathrm{Ra}\end{array}\right]=\left[\begin{array}{c}0 \\ 0 \\ 0 \\ 0 \\ 0 \\ \mathrm{Ra}_{0}\end{array}\right]+\epsilon\left[\begin{array}{c}A_{11} \\ B_{11} \\ B_{21} \\ C_{11} \\ C_{21} \\ 0\end{array}\right]+\epsilon^{2}\left[\begin{array}{c}A_{12} \\ B_{12} \\ B_{22} \\ C_{12} \\ C_{22} \\ \mathrm{Ra}_{2}\end{array}\right]+\epsilon^{3}\left[\begin{array}{c}A_{13} \\ B_{13} \\ B_{23} \\ C_{13} \\ C_{23} \\ 0\end{array}\right]+\cdots$

Substituting (34) in Eq. (30) and comparing like powers of $\epsilon$ on either side of the resulting equations, we get systems of different orders. 


\subsection{First-order system}

$\mathcal{L} \mathbf{v}_{\mathbf{1}}=0$,

where

$\mathbf{v}_{\mathbf{1}}=\left[\begin{array}{lllll}A_{11} & B_{11} & B_{21} & C_{11} & C_{21}\end{array}\right]^{\operatorname{Tr}}$.

$\mathcal{L}=\left[\begin{array}{ccccc}-\delta^{2} \operatorname{Pr} & -\frac{\pi \operatorname{Pr} \sqrt{\mathrm{Ta}}}{\delta} & 0 & k \operatorname{PrRa}_{0} \xi_{1} & 0 \\ \pi \operatorname{Pr} \sqrt{\mathrm{Ta}} & \delta^{2} \operatorname{Pr} & 0 & 0 & 0 \\ 0 & 0 & -4 k^{2} \operatorname{Pr} & 0 & 0 \\ k & 0 & 0 & -\delta^{2} & 0 \\ 0 & 0 & 0 & 0 & -4 \pi^{2}\end{array}\right]$

The condition for nontrivial solution of the homogeneous linear system (35) yields

$R a_{0}=R a_{s}$

and the solution to the system (35) is given by

$\mathbf{v}_{\mathbf{1}}=\left[\begin{array}{lllll}A_{11} & \frac{\pi \sqrt{T a}}{\delta^{2}} A_{11} & 0 & \frac{k}{\delta^{2}} A_{11} & 0\end{array}\right]^{T r}$.

\subsection{Second-order system}

$\mathcal{L} \mathbf{v}_{2}=\mathcal{N}_{2}$

where

$\mathbf{v}_{\mathbf{2}}=\left[\begin{array}{lllll}A_{12} & B_{12} & B_{22} & C_{12} & C_{22}\end{array}\right]^{T r}$,

and

$\mathcal{N}_{2}=\left[\begin{array}{lllll}0 & 0 & 0 & 0 & \frac{1}{2} \pi k A_{11} C_{11}\end{array}\right]^{T r}$.

The solution of Eq. (40) is

$\mathbf{v}_{\mathbf{2}}=\left[\begin{array}{lllll}0 & 0 & 0 & 0 & -\frac{k^{2}}{8 \pi \delta^{2}} A_{11}^{2}\end{array}\right]^{T r}$.

\subsection{Third-order system}

$\mathcal{L} \mathbf{v}_{\mathbf{3}}=\mathcal{N}_{3}$,

where

$\mathbf{v}_{\mathbf{3}}=\left[\begin{array}{lllll}A_{13} & B_{13} & B_{23} & C_{13} & C_{23}\end{array}\right]^{T r}$,

$$
\mathcal{N}_{3}=\left[\begin{array}{c}
\frac{d A_{11}}{d \tau}+k \operatorname{PrRa}_{0} \xi_{2} C_{11} C_{22}-k \operatorname{PrRa}_{2} \xi_{1} C_{11} \\
\frac{d B_{11}}{d \tau}+\pi k A_{11} B_{22} \\
\frac{d B_{21}}{d \tau} \\
\frac{d C_{11}}{d \tau}-\pi k A_{11} C_{22} \\
\frac{d C_{21}}{d \tau}+\frac{1}{2} \pi k\left(A_{11} C_{12}+A_{12} C_{11}\right)
\end{array}\right]
$$

We used the Fredholm alternative condition to get the Landau amplitude equation in the following form :

$Q_{1} \frac{d A_{11}}{d \tau}=Q_{2} A_{11}-Q_{3} A_{11}^{3}$

where

$Q_{1}=1-\frac{\pi^{2} \mathrm{Ta}}{\delta^{6}}+\frac{k^{2} \xi_{1} \operatorname{PrRa}_{0}}{\delta^{4}}, Q_{2}=\frac{k^{2} \xi_{1} \operatorname{PrRa}_{2}}{\delta^{2}}$,

$\left.Q_{3}=\frac{k^{4} \xi_{1} \operatorname{PrRa}_{0}}{8 \delta^{4}}-\frac{k^{4} \xi_{2} \operatorname{PrRa}_{0}}{8 \pi \delta^{4}}-\frac{\pi^{4} \mathrm{Ta}}{8 \delta^{6} \mathrm{Pr}} . \quad\right\}$

It is now well known that there is a clear analogy between electro-convection and ferro-convection (see Siddheshwar [20]). To reiterate this in the context of the present problem, we first write down the dimensionless governing equations of rotating electro-convection by following Siddheshwar and Radhakrishna [46] in applying an AC electric field and by incorporating Coriolis force as done in Sect. 3. We get the dimensionless governing equations in the form:

$\boldsymbol{\nabla} \cdot \mathbf{q}=0$,

$\frac{1}{\operatorname{Pr}}\left[\frac{\partial \mathbf{q}}{\partial t}+(\mathbf{q} \cdot \nabla \mathbf{q})\right]=-\nabla P+\operatorname{Ra}(1+L) \theta \hat{\mathbf{k}}$

$$
-\operatorname{Ra} L \phi_{z} \hat{\mathbf{k}}+\nabla^{2} \mathbf{q}+\sqrt{\operatorname{Ta}}(\mathbf{q} \times \hat{\mathbf{k}})-\operatorname{Ra} L \theta \boldsymbol{\nabla} \phi_{z^{\prime}}
$$

$\frac{\partial \theta}{\partial t}+(\mathbf{q} \cdot \boldsymbol{\nabla}) \theta=\nabla^{2} \theta+w$,

$\frac{\partial^{2} \phi}{\partial x^{2}}+\frac{\partial^{2} \phi}{\partial z^{2}}-\frac{\partial \theta}{\partial z}=0$

In Eqs. (49)-(52), the quantity $\phi$ is now the electric potential and $L$ is the electric number. It is quite clear that Eqs. (49)-(52) can be obtained from Eqs. (21)-(24) by replacing $M_{1}$ and $M_{3}$ by $L$ and unity, respectively. Using this 
one-to-one correspondence between the ferro-convection and the electro-convection problems, we can easily obtain the Rayleigh numbers of the linear stability, weakly nonlinear stability, and energy stability analyses
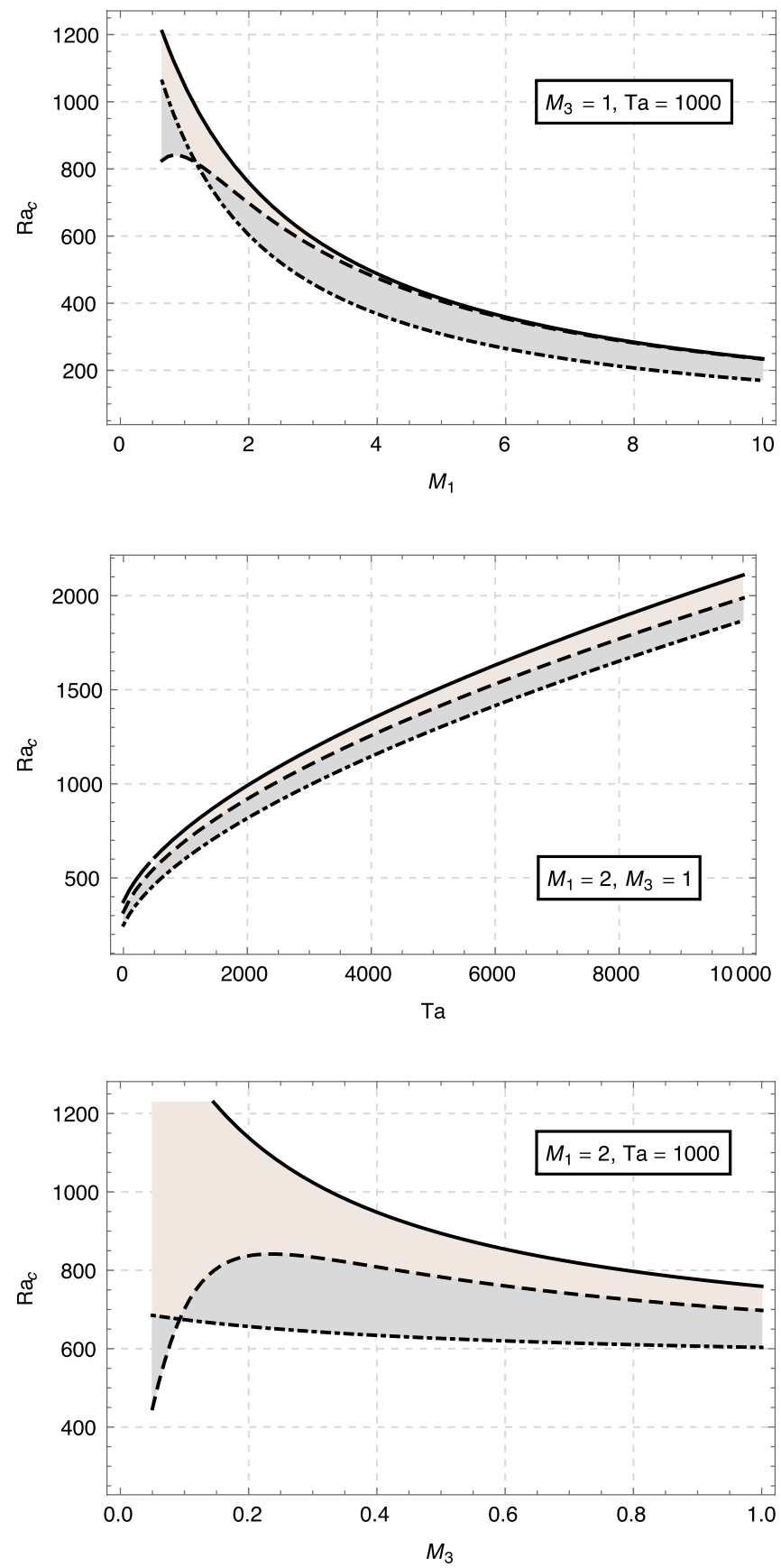

Fig. 2 Comparison of the critical Rayleigh and wave numbers for the rotating ferro-convection problem. The solid lines (-), dashed lines $(--)$, and the dot-dashed lines (- - $)$ correspond to the results obtained by linear stability, weakly nonlinear stability, and energy stability, respectively. The shaded region between the solid lines ( - ) and dashed lines (- - ) in the critical Rayleigh of rotating electro-convection by using Eqs. (32), (31), and (33), respectively.

With the necessary groundwork prepared thus far on the study pertaining to rotating free and
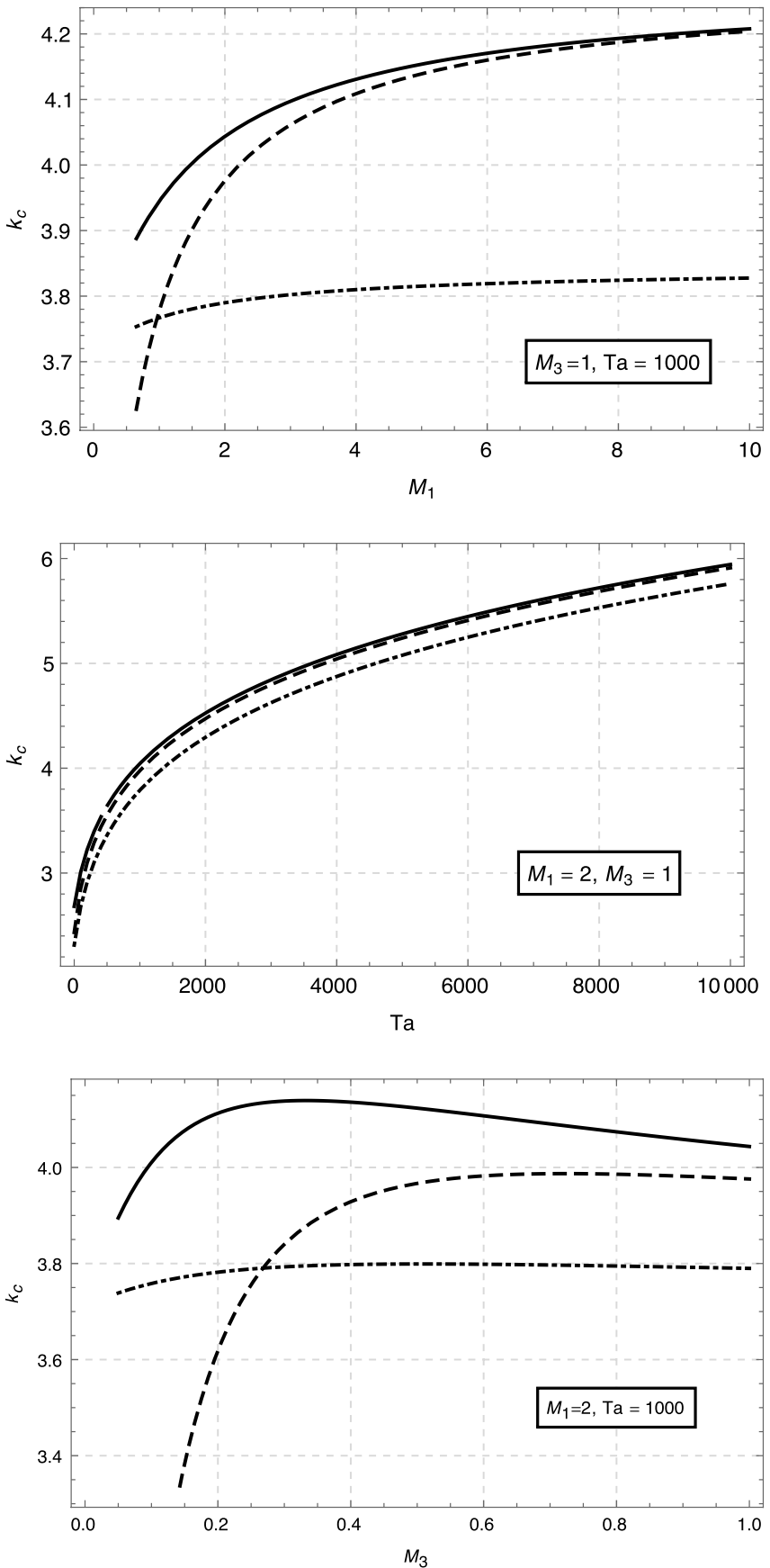

number plots represents the subcritical region due to weakly nonlinear stability analysis and the shaded region between the dashed lines (- - ) and the dot-dashed lines (-- $)$ in the critical Rayleigh number plots represents the subcritical region due to energy stability 
electro-convection, we move on to the results of the study and the discussion of it after noting that the results on

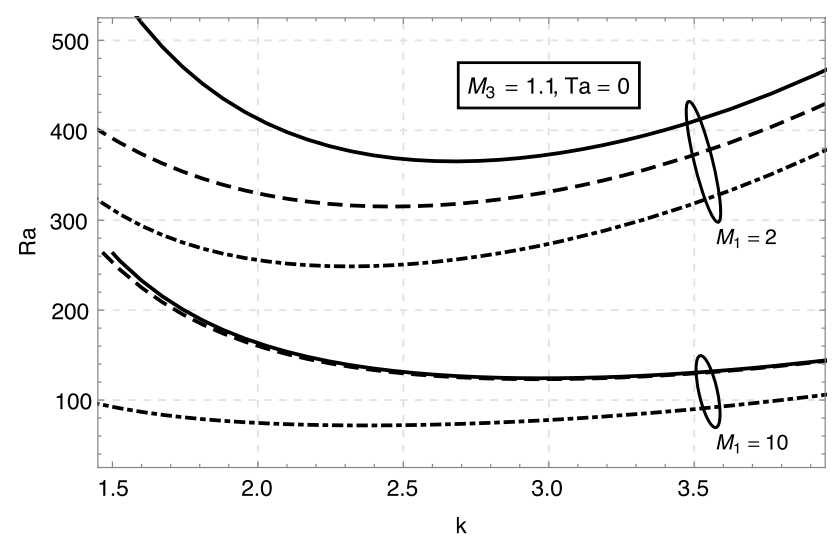

Fig. 3 Comparison of the Rayleigh numbers vs. wave number plots obtained by (-) linear stability [16], (- - ) weakly nonlinear (present study), and (- - ) energy stability [43] for $\mathrm{Ta}=0$
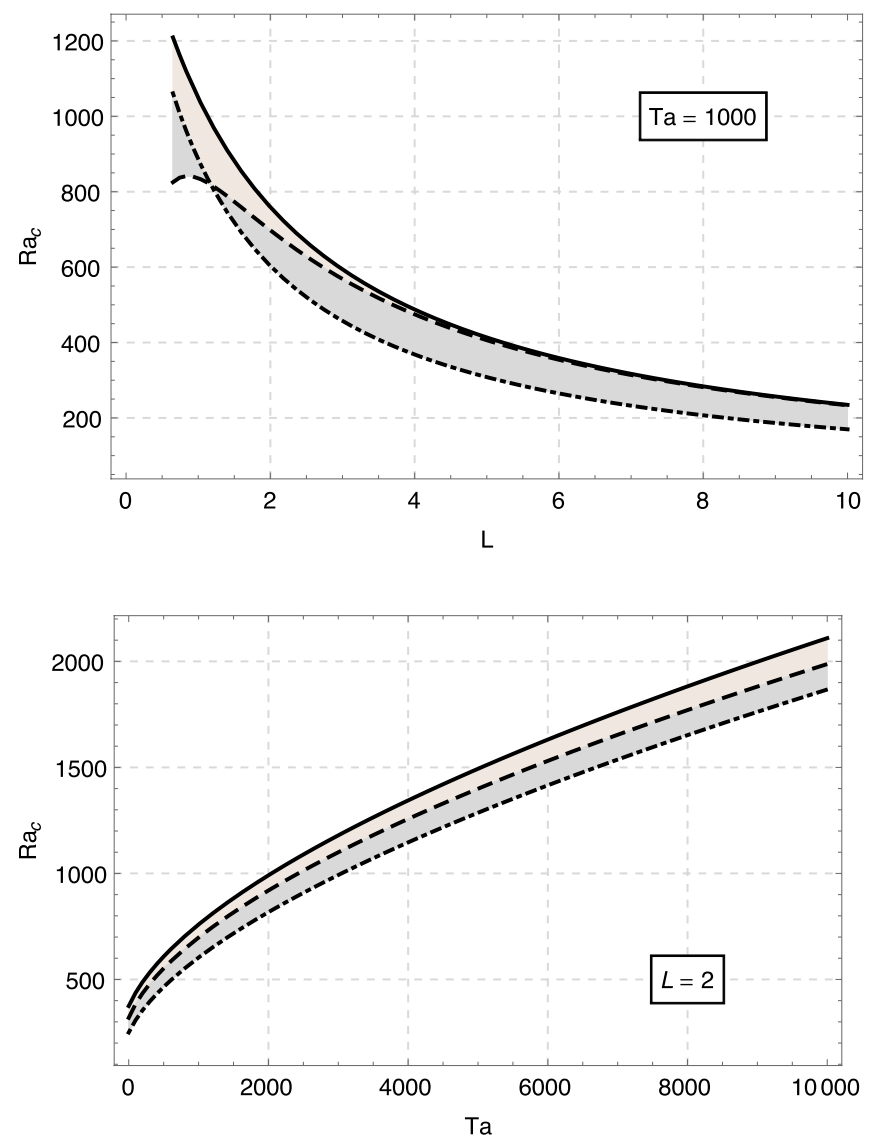

Fig. 4 Comparison of the critical Rayleigh and wave numbers for the rotating electro-convection problem. The solid lines (-), dashed lines ( --$)$, and the dot-dashed lines (- - $)$ correspond to the results obtained by linear stability, weakly nonlinear stability, and energy stability, respectively. The shaded region between the solid lines ( - ) and dashed lines (- -$)$ in the critical Rayleigh rotating ferro-convection are available in the following cases:

1. linear stability analysis [40] and

2. energy stability analysis [43].

The corresponding works on rotating electro-convection are not available.

\section{Results and discussion}

In the first part of the paper, we present a weakly nonlinear stability analysis and this yields a critical Rayleigh number which throws light on the possibility of subcritical motions. Comparison of the critical Rayleigh number and the wave number of the linear, weakly nonlinear, and energy stability theories is made for different values of the
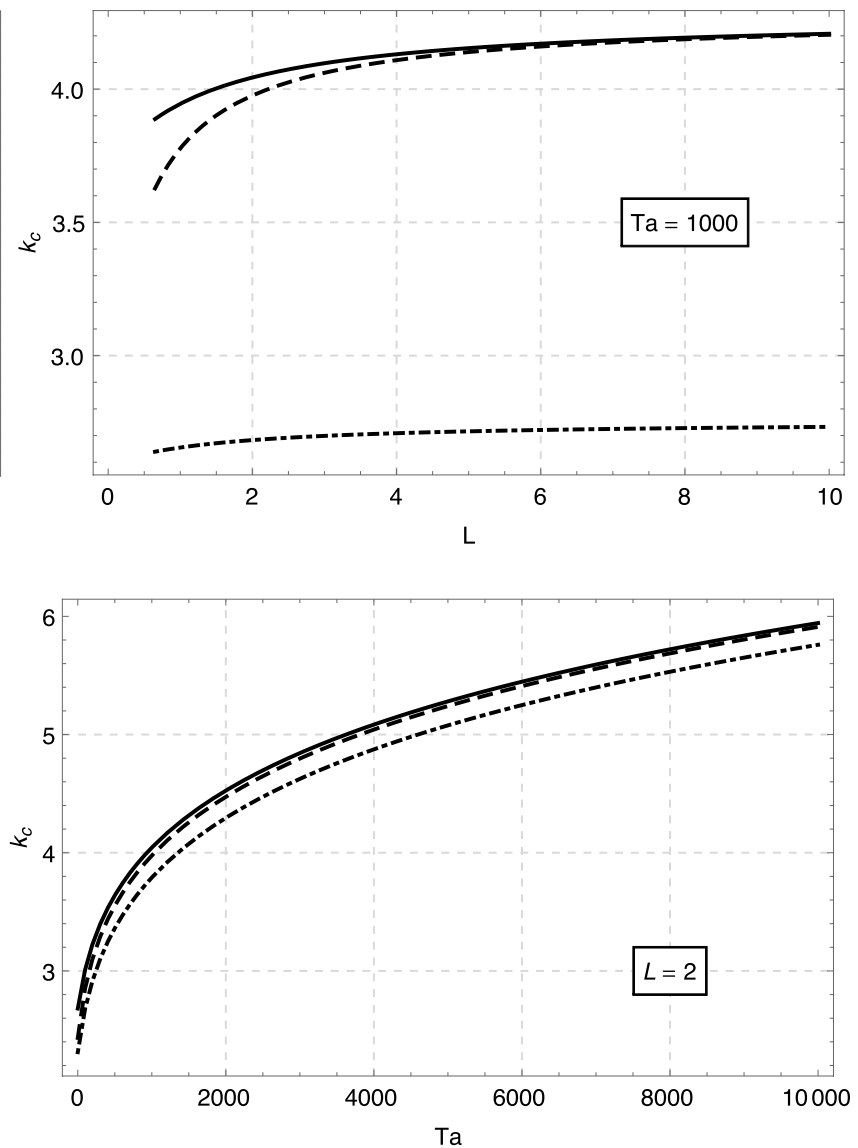

number plots represents subcritical region due to weakly nonlinear stability analysis and the shaded region between the dashed lines $(--)$ and the dot-dashed lines (- - ) in the critical Rayleigh number plots represents the subcritical region due to energy stability 
parameters of the problem. These results are presented in Fig. 2.

From the figure, it is obvious that for the present problem subcritical motions are possible. Further, it is apparent that the energy stability analysis yields the least critical Rayleigh number. A look at the critical wave number plot in Fig. 2 reveals that for the considered range of parameter values, the critical wave number varies in tandem with its corresponding Rayleigh number.

The comparison of the present results for the case Ta $=0$, with the linear stability analysis of Finlayson [16] and with the energy stability analysis of Sunil and Mahajan [43], is depicted in Fig. 3. The possibility of subcritical motions can be observed using the finite-amplitude analysis for smaller values of the buoyancy magnetization parameter $M_{1}$, whereas for larger values of $M_{1}$ the linear and finiteamplitude analysis predicts approximately the same critical Rayleigh number.

In the second part of the paper, the governing equations for the rotating electro-convection problem are shown to be obtainable from the corresponding problem of rotating ferro-convection. For most values in the considered range of parameters' values, the result of the two problems is qualitatively similar. This result is depicted in Fig. 4.

The analytically intractable fifth-order Lorenz model with two quadratic nonlinearities for the rotating ferro/ electro-convection problems is reduced to the analytical tractable Landau model with cubic nonlinearity using the method of multi-scales. The analytical solution of the Landau equation in the case of a representative rotating ferro-convection problem is presented for the following two cases:

1. Linearized Landau equation and

2. The cubic Landau equation.

We observed from Fig. 5 that in the absence of the cubic nonlinearity the solution of the Landau equation blows up in time while in its presence the solution remains bounded. This is a feature of the solution of the original Lorenz model and is preserved by the reduced Landau
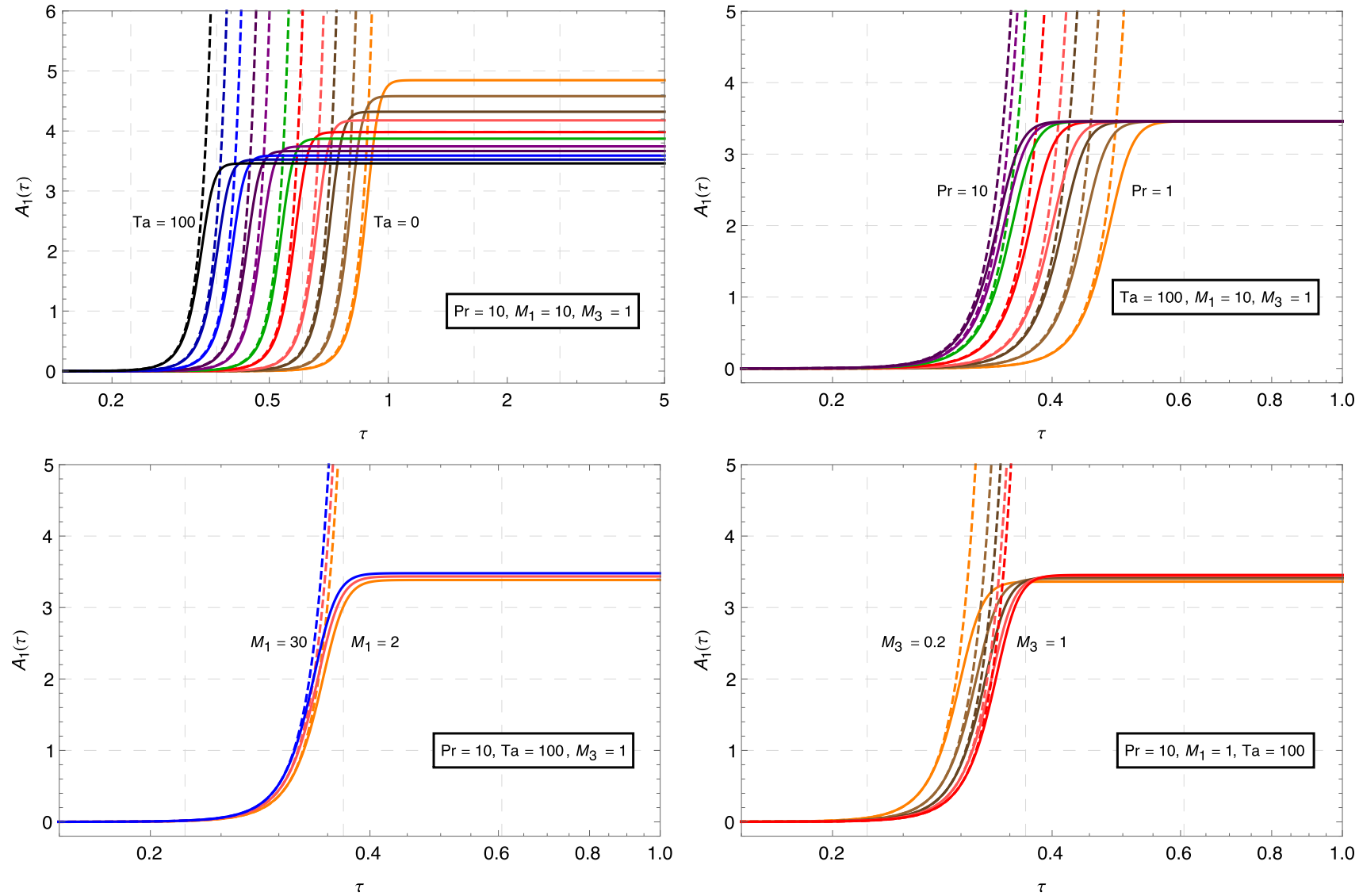

Fig. 5 The time-series plot of the amplitude of convection. The dashed lines (- - ) correspond to the exact solutions of the linearized form of Landau equation and solid lines (-) correspond to the exact solutions of Landau equation 
equation. Computation reveals that the nature of the solution of the rotating ferro-convection problem is also seen in the rotating electro-convection problem.

\section{Conclusion}

1 The present study leads to the conclusion that the critical value of the Rayleigh number of infinitesimal amplitude perturbation and those by the energy stability and weakly nonlinear stability analyses are not the same. The latter two instabilities are, in fact, subcritical in nature. These results clearly point to the fact that in so far as rotating Rayleigh-Bénard convection in ferromagnetic liquids or dielectric liquids is concerned, the linear stability analysis is inadequate and so is the weakly nonlinear stability analysis.

2 The present paper is a good example of treating rotating ferro-convection and electro-convection in a unified way. This would mean that there is really no need to study the electro-convection in isolation as the results can be got from the ferro-convection problem by considering the electric number in place of the buoyancy magnetization parameter and by taking a value of unity for the non-buoyancy magnetization parameter.

3 The weakly nonlinear stability analysis leads to the analytically intractable five-dimensional Lorenz model (with two quadratic nonlinearities) and this can be reduced to the one-dimensional Landau model (with a cubic nonlinearity) that is analytically tractable. The latter arises due to a local nonlinear stability analysis. This procedure of reduction gives us a physically realistic bounded solution of the Landau equation and thus we thereby come to know that some results of a weakly nonlinear stability analysis can, in fact, be obtained from a local nonlinear stability analysis without the need to pursue a numerical method.

Acknowledgements The authors are grateful to their respective institutes of working for their encouragement. We thank the reviewer for the most valuable comments that helped us to modify the paper to the present form.

\section{Compliance with ethical standards}

Conflict of interest The authors declare that they have no conflict of interest.

\section{References}

1. Chandrasekhar S (1981) Hydrodynamic and hydromagnetic stability. Dover, New York

2. Getling AV (2001) Rayleigh-Bénard convection: structures and dynamics. World Scientific Press, Singapore

3. Bhattacharjee JK (1987) Convection and chaos in fluids. World Scientific Press, Singapore

4. Greenspan HP (1969) The theory of rotating fluids. Cambridge University Press, London

5. Veronis G (1966) Motions at subcritical values of the Rayleigh number in a rotating fluid. J Fluid Mech 24(3):545-554

6. Straughan B (2013) The energy method, stability, and nonlinear convection. Springer, Berlin

7. Rosensweig RE (1985) Ferrohydrodynamics. Cambridge University Press, Cambridge

8. Bashtovoy VG, Berkovsky BN, Vislovich AN (1988) Introduction to thermodynamics of magnetic fluids. Hemisphere, Washington

9. Nakatsuka K, Jeyadevan B, Neveu S, Koganezawa H (2002) The magnetic fluid for heat transfer applications. J Magn Magn Mater 252:360-362

10. Shliomis MI (1974) Magnetic fluids. Sov Phys Usp 17:153-169

11. Dubina SH, Wedgewood LW (2016) A Brownian dynamics study on ferrofluid colloidal dispersions using an iterative constraint method to satisfy Maxwell's equations. Phys Fluids 28:072001

12. Singh C, Das AK, Das PK (2016) Flow restrictive and shear reducing effect of magnetization relaxation in ferrofluid cavity flow. Phys Fluids 28:087103

13. Jackson BA, Terhune KJ, King LB (2017) lonic liquid ferrofluid interface deformation and spray onset under electric and magnetic stresses. Phys Fluids 29:064105

14. Hassan MR, Zhang J, Wang C (2018) Deformation of a ferrofluid droplet in simple shear flows under uniform magnetic fields. Phys Fluids 30:092002

15. Ahmed A, Fleck BA, Waghmare PR (2018) Maximum spreading of a ferrofluid droplet under the effect of magnetic field. Phys Fluids 30:077102

16. Finlayson BA (1970) Convective instability of ferromagnetic fluids. J Fluid Mech 40:753-767

17. Lalas DP, Carmi S (1971) Thermoconvective stability of ferrofluids. Phys Fluids 14:436-437

18. Schwab L, Hildebrandt U, Stierstadt K (1983) Magnetic Bénard convection. J Magn Magn Mater 39:113-114

19. Stiles PJ, Kagan MJ (1990) Thermo-convective instability of a horizontal layer of ferrofluid in a strong vertical magnetic field. J Magn Magn Mater 85:196-198

20. Siddheshwar PG (1993) Rayleigh-Bénard convection in a ferromagnetic fluid with second sound. Jpn Soc Mag Fluids 25:32-36

21. Siddheshwar PG (1995) Convective instability of ferromagnetic fluids bounded by fluid permeable magnetic boundaries. J Magn Magn Mater 149(1-2):148-150

22. Sekhar GN, Rudraiah N (1991) Convection in magnetic fluids with internal heat generation. Trans ASME J Heat Trans 113:122-127

23. Siddheshwar PG, Abraham A (2003) Effect of time-periodic boundary temperatures/body force on Rayleigh-Bénard convection in a ferromagnetic fluid. Acta Mech. 161:131-150

24. Bajaj R, Malik SK (1995) Pattern formation in ferrofluids. J Magn Magn Mater 149(1-2):158-161

25. Bajaj R, Malik SK (1997) Convective instability and pattern formation in magnetic fluids. J Math Anal Appl 207(1):172-191 
26. Blennerhassett PJ, Lin F, Stiles PJ (1991) Heat transfer through strongly magnetized ferrofluids. Proc R Soc A 433:165-177

27. Abdullah AA, Lindsay KA (1991) Bénard convection in a nonlinear magnetic fluid under the influence of a non-vertical magnetic field. Continuum Mech Thermodyn 3(1):13-25

28. Zebib A (1996) Thermal convection in a magnetic fluid. J Fluid Mech 321:121-136

29. Gotoh K, Yamada M (1982) Thermal convection in a horizontal layer of magnetic fluids. J Phys Soc Jpn 51:3042-3048

30. Luo WL, Du T, Huang J (1999) Novel convective instability in a magnetic fluid. Phys Rev Lett 82(20):4134-4137

31. Russell CL, Blennerhassett PJ, Stiles PJ (1999) Supercritical analysis of strongly nonlinear vortices in magnetized ferrofluids. Proc R Soc Lond A 455:23-67

32. Schwab L, Stierstadt K (1987) Field-induced wavevector-selection by magnetic Bénard-convection. J Magn Magn Mater 65(2-3):315-316

33. Schwab $L$ (1990) Thermal convection in ferrofluids under a free surface. J Magn Magn Mater 85(1-3):199-202

34. Schwab L, Hildebrandt U, Stierstadt K (1983) Magnetic Bénard convection. J Magn Magn Mater 39(1-2):113-114

35. Sunil Mahajan A (2008) A nonlinear stability analysis for magnetized ferrofluid heated from below. Proc R Soc A 464:83-98

36. Tangthieng C, Finlayson BA, Maulbetsch J, Cader T (1999) Heat transfer enhancement in ferrofluids subjected to steady magnetic fields. J Magn Magn Mater 201:252-255

37. Snyder SM, Cader T, Finlayson BA (2003) Finite element model of magnetoconvection of a ferrofluid. J Magn Magn Mater 262:269-279

38. Laroze D, Siddheshwar PG, Pleiner H (2013) Chaotic convection in a ferrofluid. Commun Nonlinear Sci Numer Simul 18(9):2436-2447

39. Rahman H, Suslov SA (2015) Thermomagnetic convection in a layer of ferrofluid placed in a uniform oblique external magnetic field. J Fluid Mech 764:316-348

40. Gupta MD, Gupta AS (1979) Convective instability of a layer of ferromagnetic fluid rotating about a vertical axis. Int J Eng Sci 17:271-277
41. Venkatasubramanian S, Kaloni PN (1994) Effects of rotation on the thermoconvective instability of a horizontal layer of ferrofluids. Int J Eng Sci 32:237-256

42. Aurenhammer GK, Brand HR (2000) Thermal convection in a rotating layer of a magnetic fluid. Eur Phys J B 16:157-168

43. Sunil Mahajan A (2008) A nonlinear stability analysis for rotating magnetized ferrofluid heated from below. Appl Math Comput 204:299-310

44. Kaloni PN, Lou JX (2004) Weakly nonlinear instability of a ferromagnetic fluid rotating about a vertical axis. J Magn Magn Mater 284:54-68

45. Nanjundappa CE, Shivakumara IS, Prakash HN (2014) Effect of Coriolis force on thermomagnetic convection in a ferrofluid saturating porous medium: a weakly nonlinear stability analysis. J Magn Magn Mater 370:140-149

46. Siddheshwar PG, Radhakrishna D (2012) Linear and nonlinear electro-convection under AC electric field. Commun Nonlinear Sci Numer Simul 17:2883-2895

47. Siddheshwar PG (2002) Ferrohydrodynamic and electrohydrodynamic instability in Newtonian liquids: an analogy. East West J Math Spec Vol Comput Math Model 2:143-146

48. Siddheshwar PG (2002) Oscillatory convection in ferromagnetic, dielectric and viscoelastic liquids. Int J Mod Phys B 16:2629-2635

49. Siddheshwar PG, Kanchana C (2017) Unicellular unsteady Rayleigh-Bénard convection in Newtonian liquids and Newtonian nanoliquids occupying enclosures: new findings. Int J Mech Sci 131-132:1061-1072

50. Dey P, Suslov SA (2016) Thermomagnetic instabilities in a vertical layer of ferrofluid: nonlinear analysis away from a critical point. Fluid Dyn Res 48(6):061404

51. Suslov SA (2008) Thermomagnetic convection in a vertical layer of ferromagnetic fluid. Phys Fluids 24:084101

Publisher's Note Springer Nature remains neutral with regard to jurisdictional claims in published maps and institutional affiliations. 